\title{
REAL TIME ONLINE PROFILE MEASUREMENT SYSTEM FOR STEEL WIRE PRODUCTS
}

\author{
Saurabh ${ }^{1}$, Rajiv RANJAN ${ }^{2}$, Chitresh KUNDU ${ }^{3}$, Jayendra KUMAR ${ }^{4}$, Prabal PATRA ${ }^{5}$ \\ ${ }^{1}$ National Institute of Technology, Dept. of ECE, Jamshedpur, India, email: saurabhkj93@ gmail.com \\ ${ }^{2}$ National Institute of Technology, Dept. of ECE, Jamshedpur, India, email: rajiv210691@ gmail.com \\ ${ }^{3}$ Tata Steel, Instrumentation \& Control, email: chitresh.kundu@ tatasteel.com \\ ${ }^{4}$ National Institute of Technology, Dept. of ECE, Jamshedpur, India, jkumar.ece@ nitjsr.ac.in \\ ${ }^{5}$ Tata Steel, Instrumentation \& Control, email: prabal.patra@ tatasteel.com
}

\begin{abstract}
The quality specifications for products such as wire rods produced by the steel industry are becoming ever more demanding. This demand has led to achieve uniform product quality at high production speeds. As a result, there is a need for the systems that operate in real time and continuously measure cross-section profile of the rods and detect if any change or deviation from the desired behaviour in their cross-section. To reach this goal, a laser-based computer-controlled technology provides a useful solution. In this paper, a high speed, low cost and high accuracy non-contact laser-based measuring system has been proposed and developed using a STM32 microcontroller. The system can measure the thickness of solid, non-transparent wire rods using the linear charged-coupled sensor and sent to a microcontroller unit to further analyse and continuously display the cross-sectional profile in real time. Any deviation in the dimension over the length of the wire along with its position with respect to starting of the wire can be detected in real time. The designed and developed system has capability of measurement accuracies and respectabilities to the micrometre level.
\end{abstract}

Keywords: Laser Micrometer, Slip Rings, Reflective Sensor, ARM Microcontroller, Radio Frequency Module

\section{INTRODUCTION}

Steel industries operate mill rods all over the world. They form a major portion of their overall production. The production spectrum consists of ball bearing steel, valve steel, wire rods, grids etc.

The customers of steel industry require these products with tight dimensional accuracy as these are widely used in other industries and in homes. Key dimensions of the rods such as height, width, shoulder sizes and out-of-round profile must be available in real-time to control the high-volume rolling process efficiently and to comply with quality assurance requirements. Neither width and height nor over- or underfill could be either displayed by the existing oscillating single-axis gauges. Therefore, manual cutting, and measurement of samples still had to be done. Furthermore, the entire coils could not be represented by these samples. Hence a new system for profile measurement became necessary. Any deformity or deviation from actual parameter results in rejection of products by the customers which cause high losses to the manufacturer.

The diameter range of wire rods varies from 5 to $16 \mathrm{~mm}$. These are continuously produced at the rate of $100 \mathrm{~m} / \mathrm{s}$. Now a day's wire rods are measured with hand held devices or through diameter gauges. These methods of visual inspection are less efficient and prone to human errors. Moreover, it is time consuming and generally cannot cope up with the high-speed production.

Diameter and profile along the entire length of the billet should be determined by the new profile measurement system. Optimal crop cuts at head and tail of the billets is allowed by this. Now a days there is trend towards smaller production lots. It results in frequent change in sizes and steel grades. Hence, rod diameter and profile need to be displayed in real time. This helps in quickly adjusting the mill to roll within permissible tolerance. This system may help in raising the dimensional accuracy of the steel rod which in turn increases the portion of good steel product. Flexibility as well as reliability is gained in the process. The system which is to be developed should have these following desired features:

- High Accuracy: Cross section profile of the wire rod should be measured with great accuracy. The error during measurement should be as less as possible, close to industry recommendations and allowed by the producer.

- High Speed: Since the production is being done at high speeds data should be processed at high speeds. The sensor should be able to send data at as high speed as possible without any delay. 
The processor should be able to calculate the results without any processing delay.

- Low cost: The system being developed should be cost effective as it needs to be installed at various places and be available to all at lower cost.

- Low maintenance: Since the system would be installed at locations where the environment is very harsh and can't be accessed daily. Hence it should require less maintenance.

- Real Time: the production speed of the wire mill rods is very high close to $100 \mathrm{~m} / \mathrm{s}$. The cross-sectional profile of the rods being produced should be available in real time. There should be a minimum delay between the displayed result and the produced wire rods. Otherwise, any defect would take a long time to be detected causing loss to the manufacturers and wastage of large amounts of resources.

Various systems have been developed which can be used to achieve these desired features. System such as optical diameter gauge [1] which uses two laser diodes for measuring the outer diameter of digital wire or designing of CCD laser for thickness measurement using microcontroller such as ARM [2] can be taken into consideration but these systems are slow and not much accurate as per demand. Some system such as LAP laser [3] for automation system uses four axis lasers for developing contours of the rods, Accuscan series [4] uses camera-based CCD laser while Meer Gauge [5] system also uses two sets of lasers for detecting contour of the wire rods. These systems developed are fast but not very much accurate as they can measure only few cross sections of the moving rods. Hence to overcome the difficulties faced by these systems we are proposing a laser micrometer based system which has been described in the next section.

\section{PROPOSED SYSTEM}

The proposed system is divided into various parts and subsequently developed into a real time embedded system which shows the contour of the moving wire rods.

\subsection{Sensor}

For measuring the profile, we are using shadowgraphy based multipurpose CCD Laser Micrometer [6]. The sensor is linearized- charged coupled device as shown in Fig.1. The sensor detects the position of a target placed between the receiver and transmitter; it is possible to achieve stable target measurement due to its less sensitivity to its environment.

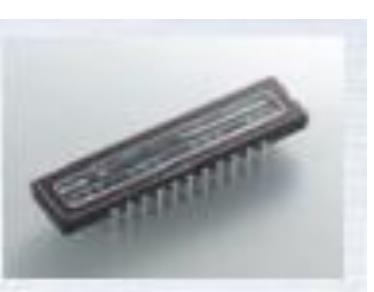

Fig. 1. Linear-CCD sensor

The transmission spot produces a patchy pattern as shown in Fig. 2 when using conventional lasers which is a laser-specific interference problem. It is caused by the laser having a single wavelength. The sensor used here solves this problem by using a multi-wavelength laser. When the sensor remains highly stable shadows are formed on the CCD clearer and even with targets that are conventionally difficult to detect such as transparent objects. The sensor can perform data processing at high speed due to the I-DSP (a parallel computing chip) incorporated in the receiver, thus reducing noise to a minimum.

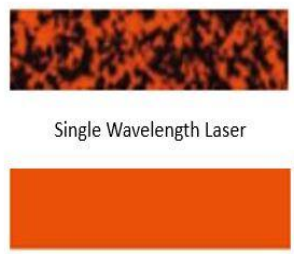

Multi Wavelength Laser

Fig. 2. Spot Image

The sensor consists of receiver and transmitter as shown in Fig. 3. The transmitter sends parallel lasers onto the receiver. A position monitor is present on the sensors which makes it possible to visually check if a target is detected.

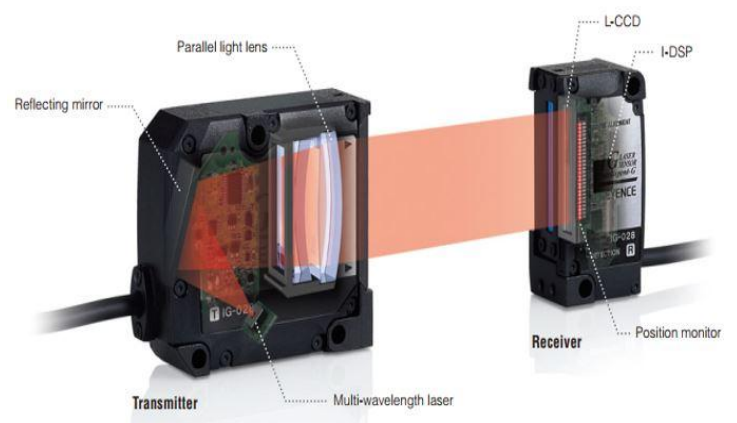

Fig. 3. Transmitter and Receiver of Sensor [6]

The amount of light received at the receiver which judges whether light is received or not can be set in this sensor which is called binarization level. Generally, a level of $25 \%$ is set to detect the outer diameter or can be changed according to use. The sensor gives the measured diameter according to the amount of light received as shown in the Fig. 4. 


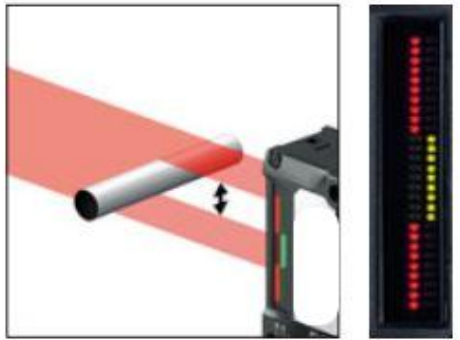

Fig. 4. Diameter measured by the sensor

The measured diameter corresponds to only one cross-section as in Fig. 5.
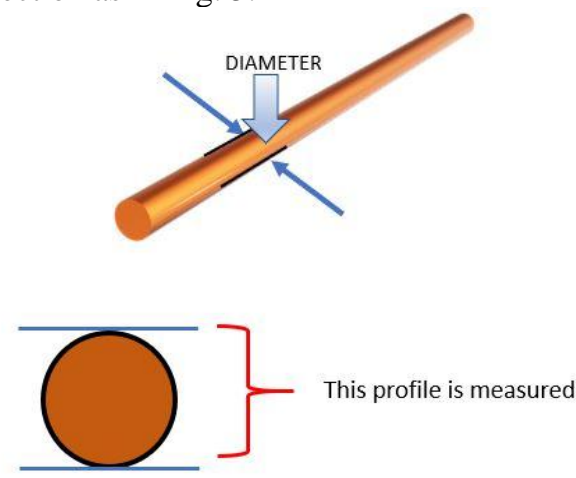

Fig. 5. Diameter of one cross section measured

\subsection{Getting complete cross section profile}

So how to get the complete cross section profile of the rod is an important question. There can be two possibilities: one such can be use of multiple sensors as used in ArcelorMittal LAP RDMS System [7] which uses six sensors placed at equal angles in rows of three system. This increases the complexity of the system and the cost.

Another possibility is using only one sensor and rotating it or the rod at high speed to take infinite cross section or as needed to successfully generate the complete profile. We cannot rotate the rod as it tends to be very long, and it is not feasible to do so. So, we have rotated the sensor in our system. The sensor is mounted on a platform and rotated at high speed as shown in Fig. 6

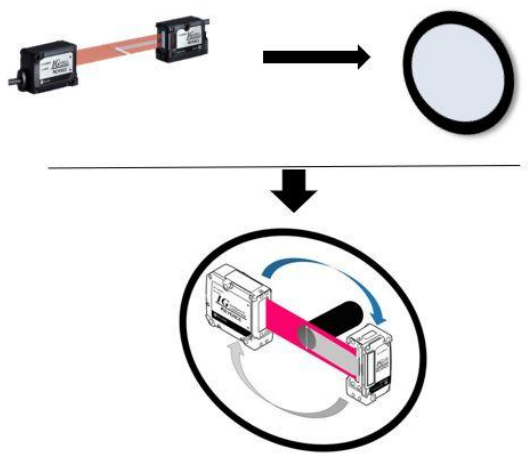

Fig. 6. Sensor mounted on a rotating platform

The sensor rotating at high speed gives multiple cross section as shown in Fig. 7. Using these multiple profiles whole cross-section of wire rods can be developed.

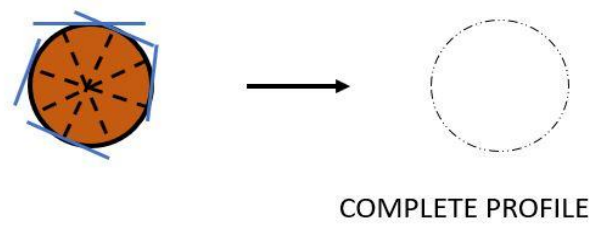

Fig. 7. Generating Complete profile

\subsection{Activating the sensor}

After mounting the sensor over the platform, we must consider two important points:

1. How to give power to the sensor?

2. How to get data from the sensor?

For supplying power to the sensor unit, we are using slip rings. The slip ring has three 3 point of contacts with the rotating assembly. Hence two can be used for supplying power i.e. VCC and Ground. Third one is used for taking data out of the sensor as shown in Fig. 8.

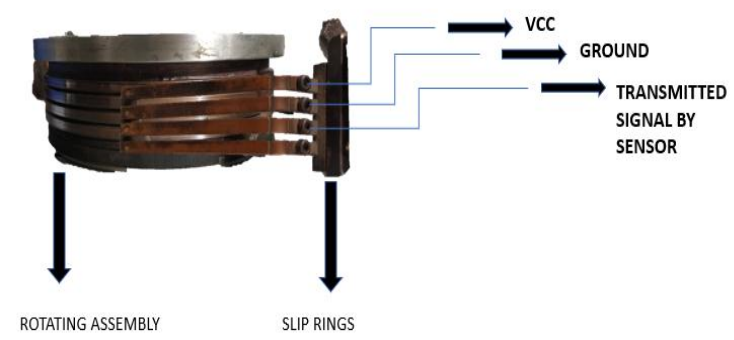

Fig. 8. Power and data out of slip rings.

When using slip rings, we must keep in consideration these three important factors [8]:

1. Bandwidth

2. EMI/EMC (Shielding)

3. Crosstalk

Bandwidth: Due to an impedance mismatch between the rings and brushes and the transmission line, a slip ring represents a discontinuity in a transmission line the extent of matching of the impedance of the brushes and the rings to the impedance of the transmission line and the effective length of discontinuity are the best indicator about the effectiveness at which the slip ring will transmit the high-speed incoming data stream. In our case data coming out of the sensor is measurement of the diameter of the wire rod. The data size taken here is 15 bytes. Hence, there is no bandwidth issue with our design.

EMI/EMC (Shielding): With regards to high speed data through slip rings Electro-magnetic interference (EMI) is one of the important performance parameters that should be kept in mind. It is important that proper shielding and grounding principles be applied at the rotational interface due to high frequency high data rate transmission lines. Specifically, a low impedance ground path should be maintained at each rotational interface. All shields should maintain their 
continuity. Proper reference level to ground should be given through the interface. Hence the data through the wire is properly shielded and grounded.

Crosstalk: Crosstalk is an important parameter when sending digital data through slip rings. Inductive coupling can have an effect in coupling of noise into sensitive signals circuits in the case of power circuits with very high switching frequencies or sharp surge spikes, i.e., very large change in input current with respect to time. However, inductive effects are almost always secondary effects and are normally handled by using care while isolating sensitive signals from power as much as possible using a physical distance.

\subsection{Receiving data from sensor continuously}

To receive data from the sensor continuously we have to give a pulse of $2 \mathrm{~ms}$ between DRQ and SG terminal [9]. For this we need to design an astable multivibrator with frequency $500 \mathrm{~Hz}$. For designing astable multivibrator we have used IC LM555N [10] precision timer and circuit diagram is shown in Fig. 9.

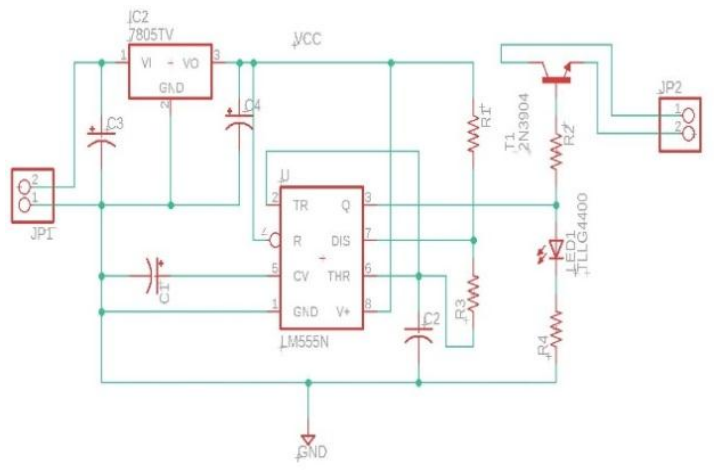

Fig. 9. LM555N TIMER Circuit
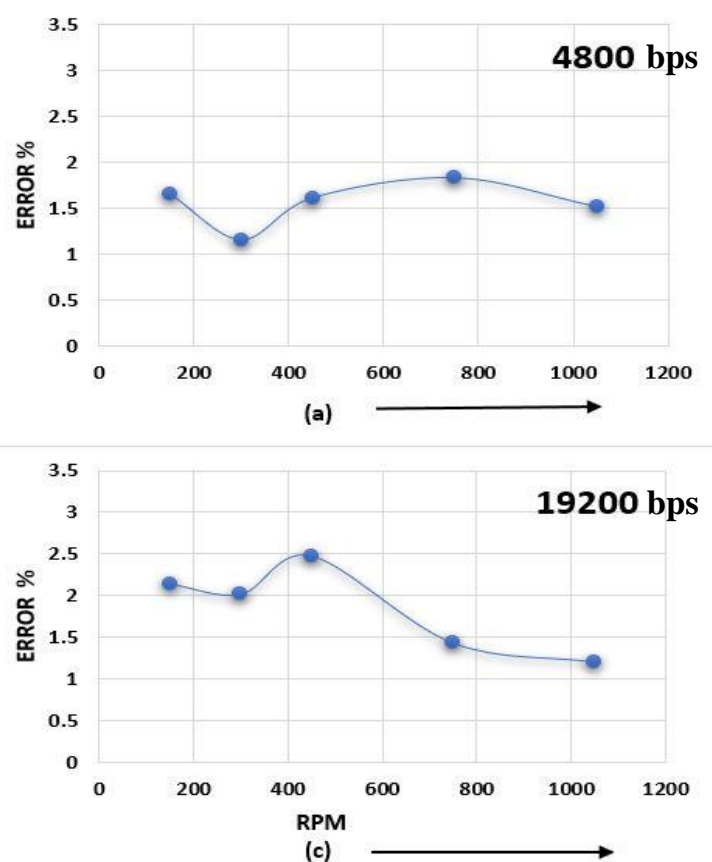

Fig. 11. Error \% at Different Baud rates (bps)

(d)
$\mathrm{R} 1=1 \mathrm{~K} \Omega, \mathrm{R} 2=2 \mathrm{~K} \Omega, \mathrm{C}=1 \mu \mathrm{F}-$ Gives pulses of $2.079 \mathrm{~ms}$ with $60 \%$ duty cycle [11]. The output data from the sensor is now received continuously. The trigger circuitry is shown in Fig. 10.

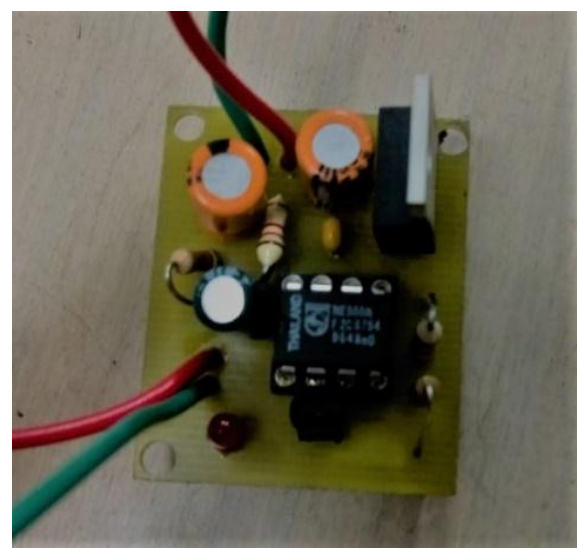

Fig. 10. LM555N TIMER PCB

\subsection{Sending data out from the rotating platform}

Data is being sent out of the sensor continuously. The data from the sensor is in RS-232 communication protocol [12]. Now the question arises how to take data out from the rotating platform. First approach is taking data out through the slip rings. the error is measured at baud rates 4800bps ,9600bps, 19200bps, 38400bps and different rotations per minute (RPM) shown in the graphs below in Fig. 11(a), (b), (c), (d).

The data coming out of the sensor is in the form of a string which is of 15 bytes in length. The string format is:

\begin{tabular}{|l|l|l|l|l|l|l|l|l|l|l|l|l|l|l|}
\hline $\mathbf{D}$ & $\mathbf{R}$ &, & $\mathbf{0}$ & $\mathbf{1}$ &, & $\mathbf{+}$ & $\mathbf{1}$ & $\mathbf{0}$ & $\cdot$ & $\mathbf{2}$ & $\mathbf{3}$ & $\mathbf{5}$ & $\mathbf{C R}$ & $\mathbf{L F}$ \\
\hline
\end{tabular}
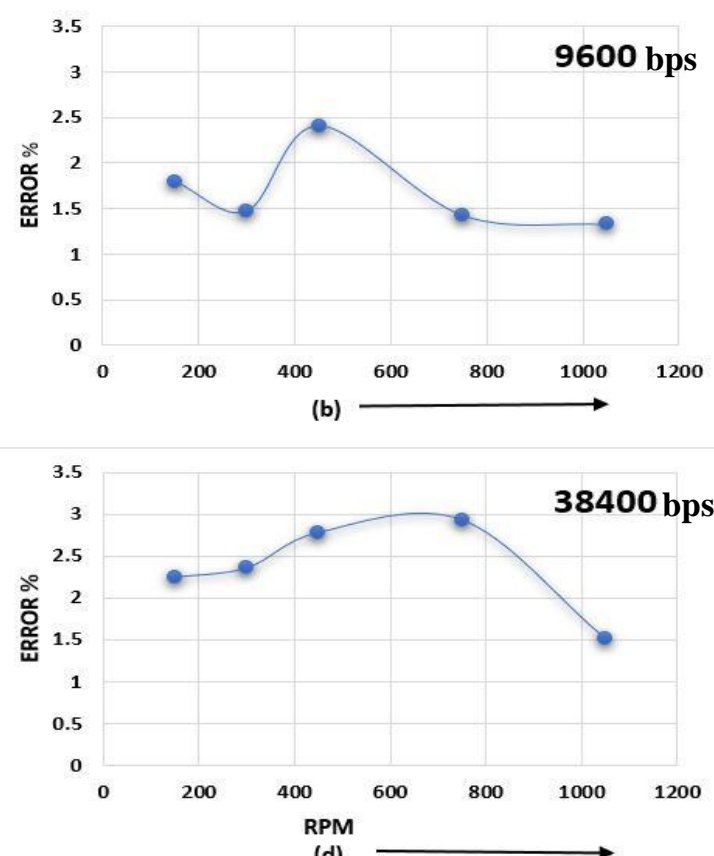
Due to errors, there are losses of frames. The string received turns out to be garbage. Hence, we cannot get correct data to get the diameter. From the observation we found that minimum error was found at $19200 \mathrm{bps}$ baud rate and $750 \mathrm{RPM}$ (rotations per minute).

\subsection{Algorithm for error checking in the string}

For checking whether the string received is correct or not, we check first byte i.e. "D" then we check the last byte i.e. "LF (Line Feed)". If both are found exact in their respective positions, then we lastly check for the $7^{\text {th }}$ byte i.e. "+". If it is also correct, then diameter value is taken out of the string, i.e. from $8^{\text {th }}$ byte to $12^{\text {th }}$ byte and used for further processing.

\subsection{Sending data out wirelessly}

We can improve the data transfer by using another approach to send data out of the rotating platform through wireless communication.

We have to use wireless RF serial link module. We cannot use any vertically or horizontally polarized antenna as it is present on a rotating platform. The waves get nullified while rotating as shown in Fig. 12.

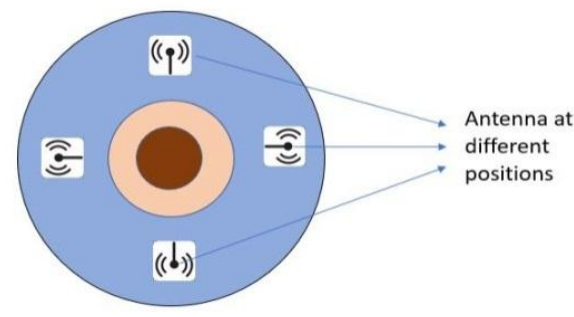

Fig. 12. Antenna on rotating platform

So, we have to use circularly polarized antenna which is a low-power and high-range wireless communication solution. This module operates in $5500-6000 \mathrm{MHz}$ frequency band. We must convert the RS-232 output of the sensor to TTL output to be fed into RF module. For this, a convertor i.e. RS232 TO TTL convertor is to be made using MAX232 IC whose circuit is shown in the Fig.13. The serial data from the sensor is sent to this module after being converted from RS-232 to TTL which is then transmitted wirelessly out of the rotating platform as shown in Fig. 14. The error is highly reduced as shown in the graph in Fig. 15.

\subsection{Detecting position of Diameter Transmitted}

The data is transmitted by the sensor, but we don't know for which position data is sent, i.e. exact location of the data. Sometimes data of same position or location is received continuously. We must know the location of the first diameter transmitted to start generating the profile as shown in Fig. 16

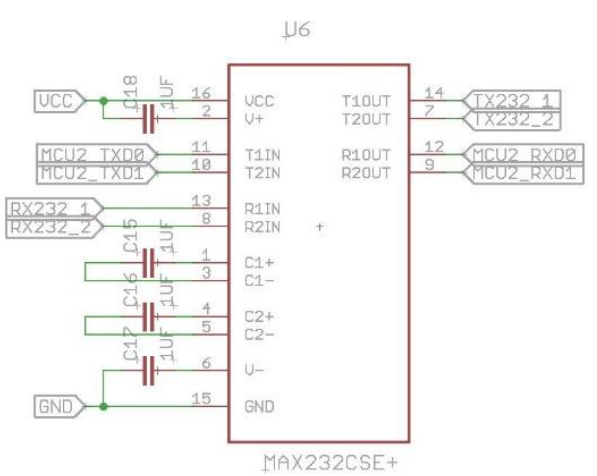

Fig. 13. RS-232 to TTL Convertor Circuit
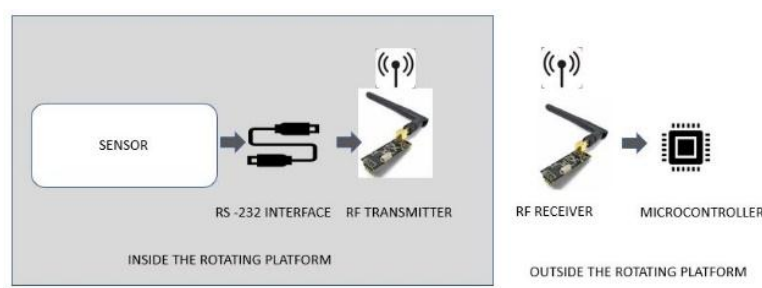

Fig. 14. Wireless Transmission of Data

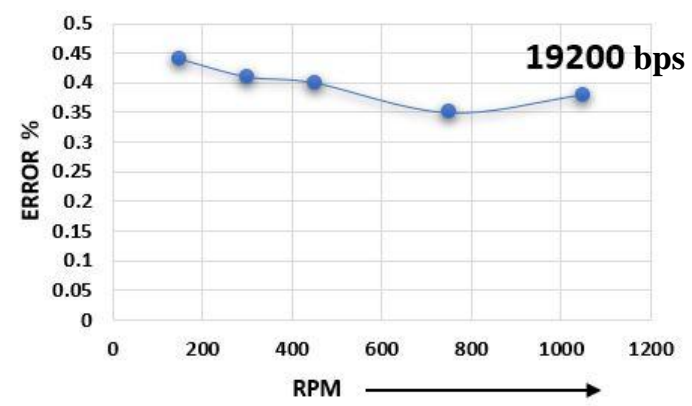

Fig. 15. Error \% vs RPM at 19200 bps

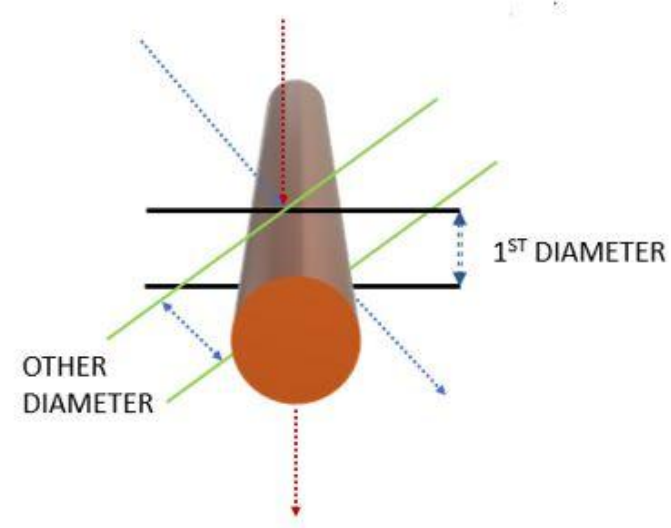

Fig. 16. Location of diameter transmitted

For detecting the position, two reflective film at 180 apart is put on the back of the rotating plate which is completely painted black as shown in Fig. 17. 


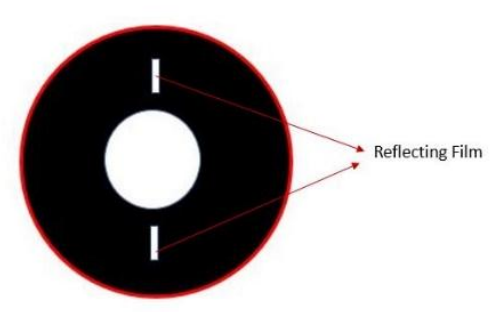

Fig. 17. Reflective film on the back of Rotating plate

A non-contact reflective object sensor OPB704 [13] is placed parallel to the reflective surface. The sensor generates an impulse when the light is reflected from the surface. When there is no reflection, no impulse or signal is generated. Fig. 18 shows position of sensor at no reflection condition.

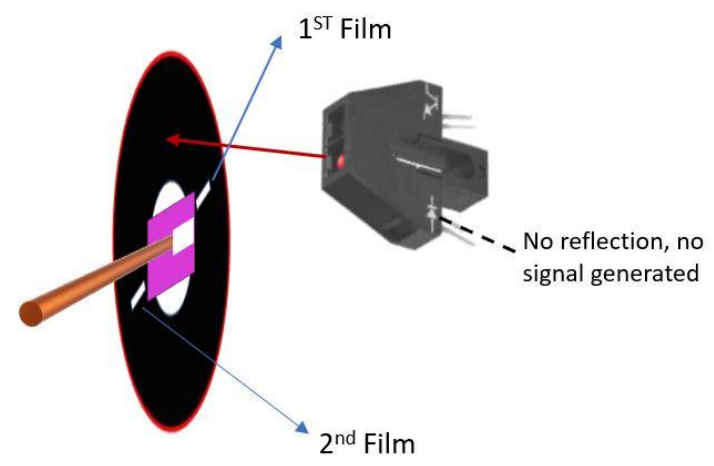

Fig. 18. No signal generated

When the sensor detects a reflecting surface, it generates the first signal which is sent to the microcontroller. The position is described in Fig. 19.

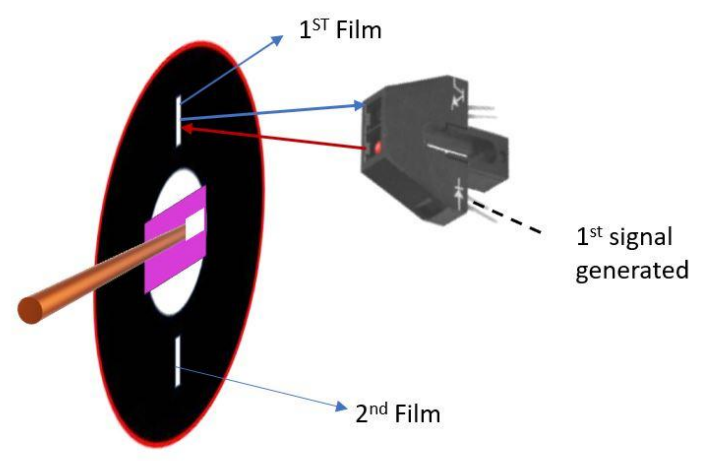

Fig. 19. $1^{\text {st }}$ signal generated

When again the sensor detects another reflecting surface, it generates another signal as shown in Fig. 20 and this process continues in every rotation.

The trigger output from the sensor is either high or low i.e. $5 \mathrm{~V}$ or $0 \mathrm{~V}$. When there is no reflecting surface the output is $5 \mathrm{~V}$ and when a reflecting surface comes, the output drops to $0 \mathrm{~V}$ as shown in Fig. 21.

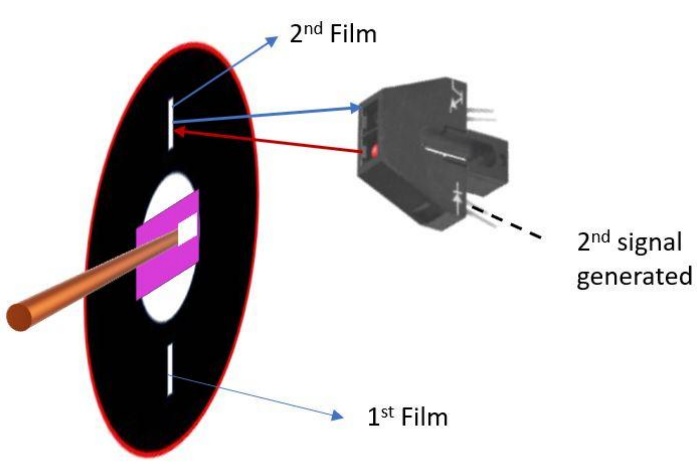

Fig. 20. $2^{\text {nd }}$ signal generated

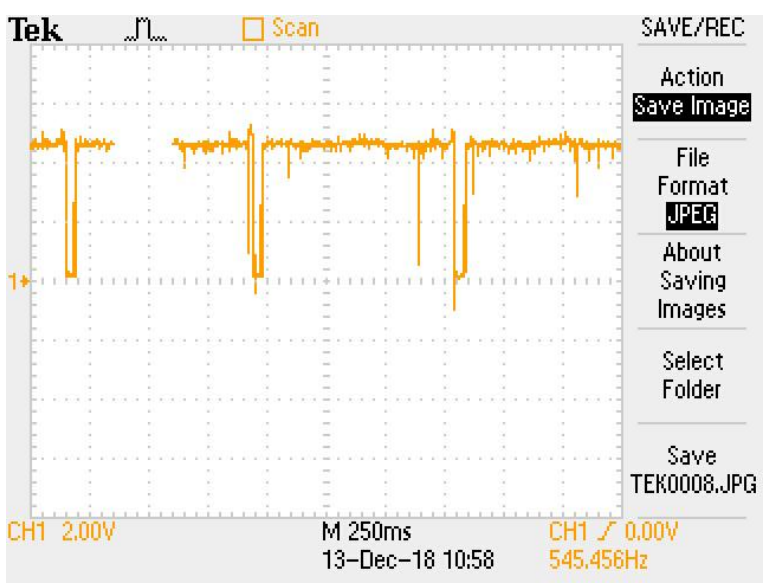

Fig. 21. Trigger output from reflective object sensor

From the output we can see that there are noises or interferences present in the signal generated by the reflective sensor. These interferences cause incorrect interrupt response at the microcontroller side.

\subsection{Noise Elimination in Signal Generated by Reflective sensor}

For eliminating the noise from the triggered output of the reflective object sensor we have used differential signalling. A pair of differential signal convertors are used [14]. The input is fed into one differential signal convertor. The output from the differential pair pin is taken out and fed into another differential signal convertor. The noise gets eliminated from the signal generated by the reflective sensor. The circuit is described in Fig.22.

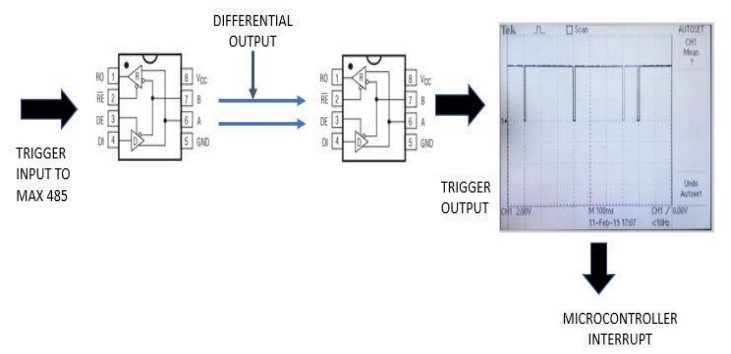

Fig. 22. Noise Elimination using Differential Signalling 
The output with no noise is shown in Fig. 23

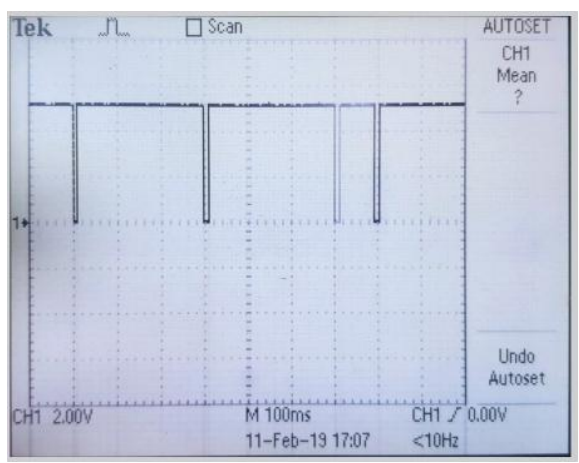

Fig. 23: Noiseless Output

\subsection{Generation of Output}

The data from the sensor and signal generated by the reflective sensor is sent to a microcontroller unit. There are many microcontrollers available in the market starting from primitive 8051 [15] to AVR series [16] and ARM microcontrollers [17]. We have used ARM microcontroller F1 seriesSTM32F103C8 [18] which is a 32-bit microcontroller.

The signal generated by the reflective sensor is sent to the interrupt terminal of the microcontroller. As shown in Fig. 18 when there is no signal generation, microcontroller does not receive data from the receiving antenna which is sending string containing the diameter. When $1^{\text {st }}$ signal is generated as shown in Fig. 19, microcontroller receives an interrupt and it starts storing the strings into its receiving buffer. It also checks whether the received strings are correct or not as we have checked previously. Time between two signal generation is used to calculate the frequency or rotation speed. The diameter is taken from the correct strings and sent along with frequency in the form of a string to MATLAB where the polar plot is created in real time.

\section{EXPERIMENTAL OBSERVATION}

The system diagram can be seen in Fig. 24.

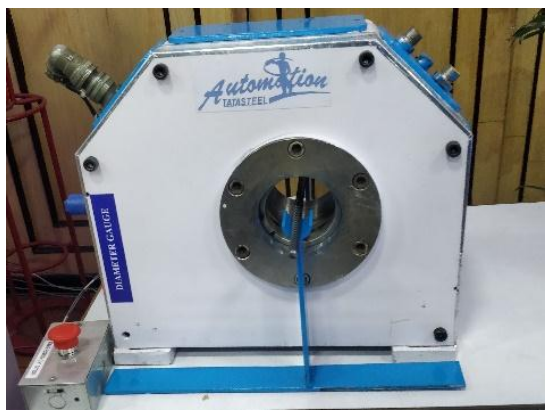

Fig. 24. Actual System

Three specimens of rods are taken, and their respective output are shown below in Fig. 25, 26, 27.

Mean radius gives the mean of all the radius of the cross section measured in one rotation denoted by $\mu$.
Circularity Factor $(C F)$ tells the standard deviation from the mean radius. The closer it is to zero more the circular shape of the rod is. It is given by equation (1).

$$
C F=\sqrt{\frac{\sum_{1}^{N}(r-\mu)^{2}}{N}}
$$

Where $r=$ radius at a position

$N=$ Total number of positions in one rotation. $\mu=$ Mean Radius for one rotation

the profile is generated at high speed by taking at least 120 points for the whole cross-section, hence the profile seems almost cylindrical in some cases.

\section{Specimen 1:}

\section{Perfect cylindrical rod}

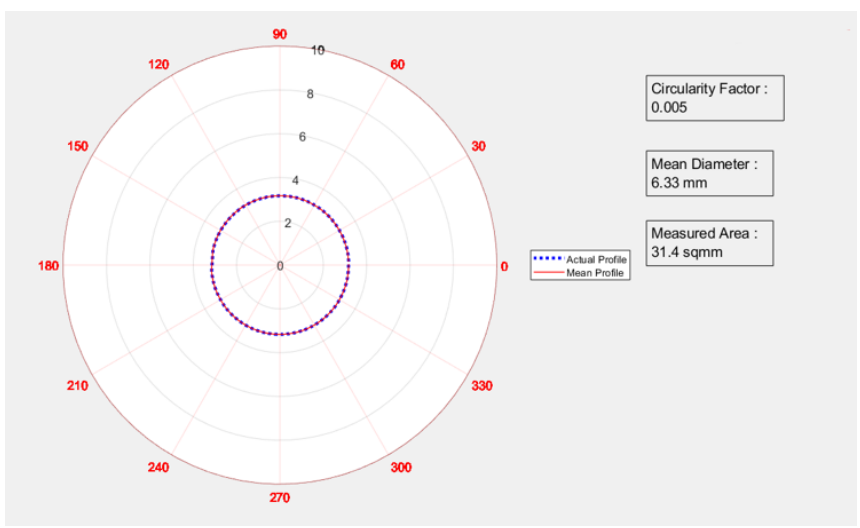

Fig. 25: Output for the Perfect cylindrical rod

Specimen 2:

TMT Bar

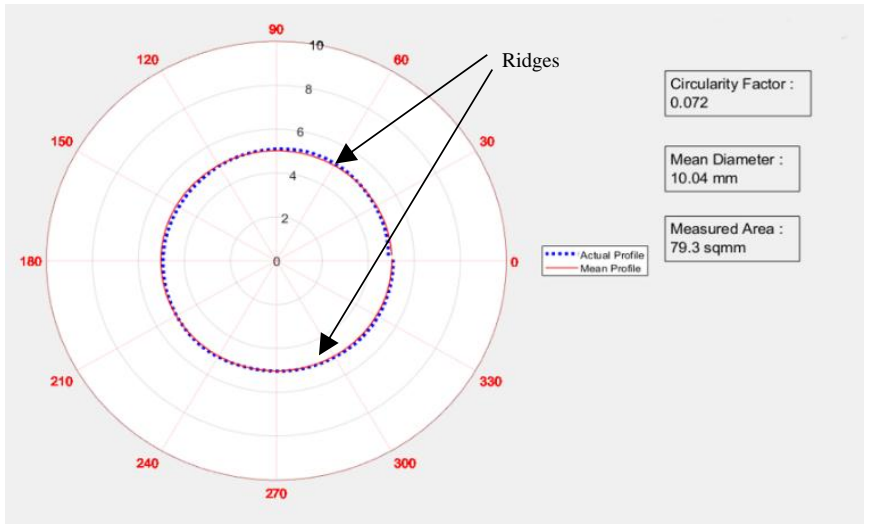

Fig. 26. Output for the TMT Bar 


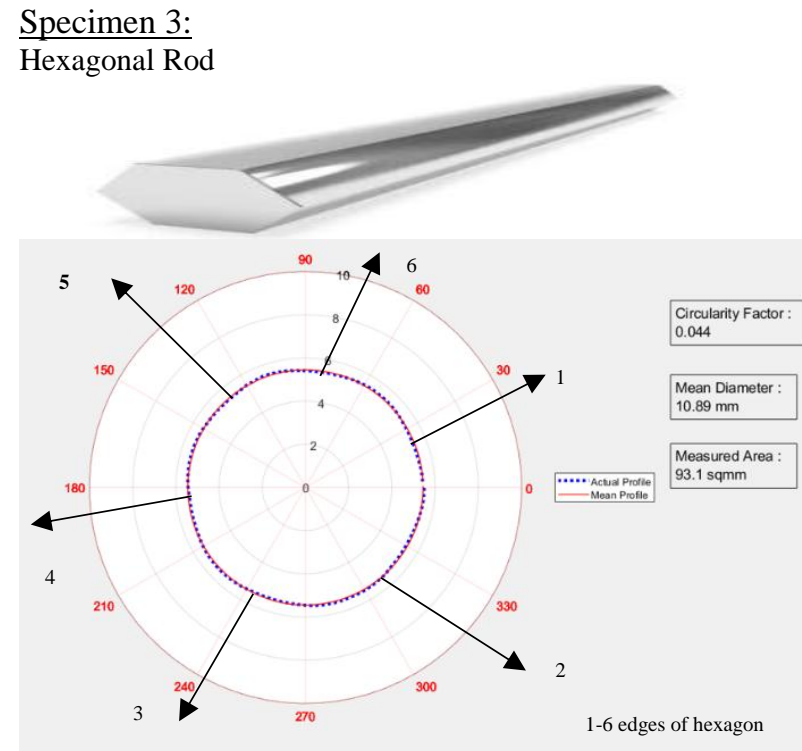

Fig. 27. Output for the Hexagonal Rod

\section{CONCLUSION}

This paper presents the development of a complete embedded system for measurement of cross-sectional profile of wire rods. It started with the calculating the diameter of a cross section of the wire rod. Then the paper gradually discusses how the complete profile could be displayed in real time to detect deviation from the desired dimension arising from the gradual increment or reduction in diameter during production. Then test results for different specimens of wire rods are shown which conclusively tells about the completion of the desired system.

\section{REFERENCES}

1. Zho $H$, Wang $X$, Wang $R$. High-speed on-line measurement of digital wire outer diameter with laser and CCD technology. Conference on Properties and Applications of Dielectric Materials, IEEE Xplore 2003.

https://doi.org/10.1109/ICPADM.2003.1218543

2. Huang Jing, Wang Yiqiang, Design of the CCD drive for Laser thickness gauge based on ARM, Proceedings of 2011 International Conference on Computer Science and Network Technology, IEEE Xplore, 2012.

https://doi.org/10.1109/ICCSNT.2011.6182454

3. LAP LASER, Measurement and Projection, July 2013.

4. Accuscan, NDC technologies, Dual- and single-axis diameter \& ovality gauges for quality- and cost-driven manufacturers. 2018.

5. SMS Group, MEERGauge- Superior profile measurement and surface quality inspection. 2016.

6. Keyence IG series Instruction manual, AS_5994_IG_C_611275_US_1028-108.

7. ArcelorMittal Hochfeld $\mathrm{GmbH}$, Measuring systems for rod and bar, Application Report, August 2017.

8. Glenn Dorsey, Application note on Critical Parameters for High Speed Data on Slip Rings. Doc No. 227, 11 January 2009.

9. DL-RS1A Communication Unit Brochure,2018.

10. Texas instruments application brochure, SNAS548D -February 2000-revised January 2015.
11. NE555 timer calculator, Available http://www.ohmslawcalculator.com/555-astablecalculator, accessed [30-Sept-2018].

12. Staszewski W, Jabłoński A, Dziedziech K. A survey of communication protocols in modern embedded condition monitoring, Diagnostyka 2018;19(2):5362. https://doi.org/10.29354/diag/86409

13. Reflective Object Sensor, TT electronics plc, General Note, Issue F, 06/2016.

14. Datasheet, MAX485, Maxim Integrated, Rev10, Sept 2014.

15. MA Mazidi, The 8051 Microcontroller, 2nd Edition 2008.

16. S. Naimi,The AVR Microcontroller and Embedded System, 1st Edition 2014.

17. AN Sloss, ARM System Developers Guide, Elsevier 2016.

18. STM32F103C8, Available https://www.st.com/en/microcontrollers/stm32f103c 8.html, accessed [18-Aug-2018].

Received 2019-06-05

Accepted 2019-09-17

Available online 2019-09-23

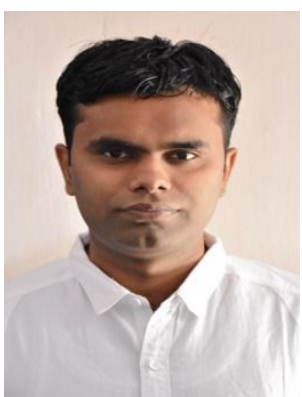

Saurabh received his $\mathrm{BE}$ degree from BIT, Mesra, Ranchi, India and M.Tech. degree from NIT, Jamshedpur, India in Embedded Systems Engineering. $\mathrm{He}$ is currently working as Test Engineer 2 (DellEMC R\&D Center). His area of research interest includes Embedded system, PCB Designing, Internet of Things, wireless communication.

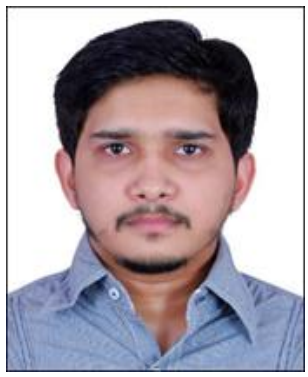

communication, IOT.

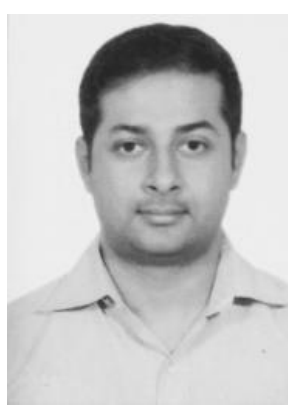

Rajiv RANJAN received his B-Tech degree from Rajasthan technical university Kota, Rajasthan, India in electronics and communication engineering and M-tech degree from NIT Jamshedpur in communication system engineering. His area of research interest includes robotics, embedded system, Antenna, wireless

Chitresh KUNDU received his M.Tech. degree from IIT Kharagpur, India, in 2010. He is currently working as Senior Technologist (Instrumentation and Control, Automation Division, TATA Steel. $\mathrm{He}$ is responsible for development of various embedded system and RADAR based application for TATA Steel. His research interests include the fields of

antennas, RADARS, embedded systems, IOT platforms. 


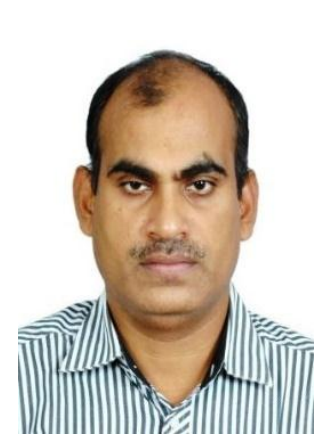

Jayendra KUMAR secured

his B.E. degree in

Electronics Engineering in the year 1995 from Nagpur University, Nagpur, India. He completed his M.E. from NIT Jamshedpur, India in 2009 and $\mathrm{Ph} . \mathrm{D}$. from Indian Institute of Technology Roorkee, India in machine learning in 2019. Presently, he is working as an Asssistant Professor in Electronics and Communication Engineering Department, National Institute of Technology Jamshedpur, India. During his long journey in academia he has guided 12 M.tech thesis, more than 40 B.Tech thesis and has also published about 40 research papers in reputed international conferences and journals. His research interests include embedded systems and computer vision specially feature extraction, classification and deep learning.

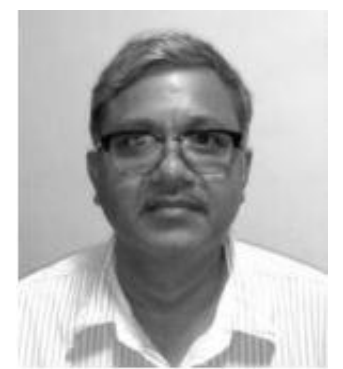

Dr Prabal PATRA completed the B.Tech. \& Ph. D in Instrumentation and Electronics engineering from the Jadavpur University, Kolkata. He is currently Head of the department of Instrumentation \& Control at Automation Division of Tata

Steel, which is a $R \& D$ department. His research areas include Control systems, Machine Vision, Embedded systems, RF \& Microwave Technology. 